Conf-9406328-.1

GA-A21733

\title{
TWO-FLUID ANALYSIS \\ OF DIMENSIONALLY SIMILAR \\ DISCHARGES
}

\author{
by \\ T.C. LUCE and C.C. PETTY
}

DECEMBER 1994 


\section{DISCLAIMER}

This report was prepared as an account of work sponsored by an agency of the United States Government. Neither the United States Government nor any agency thereof, nor any of their employees, makes any warranty, express or implied, or assumes any legal liability or responsibility for the accuracy, completeness, or usefulness of any information, apparatus, product, or process disclosed, or represents that its use would not infringe privately owned rights. Reference herein to any specific commercial product, process, or service by trade name, trademark, manufacturer, or otherwise, does not necessarily constitute or imply its endorsement, recommendation, or favoring by the United States Government or any agency thereof. The views and opinions of authors expressed herein do not necessarily state or reflect those of the United States Government or any agency thereof. 


\section{DISCLAIMER}

Portions of this document may be illegible in electronic image products. Images are produced from the best available original document. 


\title{
TWO-FLUID ANALYSIS \\ OF DIMENSIONALLY SIMILAR \\ DISCHARGES
}

\author{
by \\ T.C. LUCE and C.C. PETTY
}

This is a preprint of a paper presented at the Workshop on the Correlation between Experiment and Theory in Magnetic Confinement Systems, June 13-16, 1994, Aspenäs, Göteborg, Sweden, and to be printed in the Proceedings.

\author{
Work supported by \\ U.S. Department of Energy \\ Contract DE-AC03-89ER51114
}

GENERAL ATOMICS PROJECT 3466 DECEMBER 1994 

and, for reasonable values of $\alpha$, increases much faster with $B$ than $a$. However, there are technological limits on how much $B$ can be increased; for example, some superconductors would be limited to $17 \mathrm{~T}$ at the coil casing. Since $B$ can only be increased to a maximum amount, Eq. (4) determines the minimum size machine required to give a specified triple product. If the triple product for ignition is a factor of 10 larger than that measured on a present day machine with $a=1 \mathrm{~m}$ and $B / B_{\max }=1 / 2$, then varying $\rho_{*}$ along the dimensionless scaling path implies that the reactor must be at least $a=1.1 \mathrm{~m}$ for $\alpha=1$, $a=1.5 \mathrm{~m}$ for $\alpha=0$, and $a=4.0 \mathrm{~m}$ for $\alpha=-1$. (This assumes the ratio of toroidal field at the coil to the value at the major radius is fixed. Added geometric factors such as shielding and blankets must be factored in.) This exercise clearly shows that knowing the value of $\alpha$ sets the minimum size of a reactor without resorting to inter-machine size scaling exercises.

There are two major assumptions which must be satisfied to use this dimensionless scaling technique. First, the parametric dependence of the turbulent instabilities must remain fixed as $\rho_{*}$ is changed. If modes are stabilized or destabilized as $\rho_{*}$ changes, then clearly $F$ and perhaps even $\alpha$ will change. Second, the $\rho_{*}$ scaling must be a simple power law exponential form. There is no general principle which mandates this form; it was chosen because drift wave theories give quasilinear diffusivities of this form. Some confidence in the validity of these assumptions can be gained by testing the universality of the inferred value of $\alpha$ and by using the scaling technique to predict the performance of other present-day devices with similar configurations.

Having chosen the form of the diffusivity as in Eq. (1), theoretical constraints indicate that $\alpha$ will be in the range -1 to 1 , as mentioned above. Most theoretical transport models have a step size tied to the gyroradius and characteristic time tied to the inverse of the wave frequency, so that

$$
\chi \propto \rho_{\mathrm{s}}^{2} \omega_{*} \propto \rho_{\mathrm{s}}^{2} k_{\theta} \rho_{\mathrm{s}} \frac{c_{\mathrm{s}}}{L_{\mathrm{n}}} \propto \chi_{\mathrm{B}} \frac{\rho_{\mathrm{s}}}{a} \frac{a}{L_{\mathrm{n}}} k_{\theta} \rho_{\mathrm{s}}
$$

Making the usual assumption that $k_{\theta} \rho_{\mathrm{s}}$ is constant, Eq. (5) gives $\alpha=1$. This is called "gyro-reduced Bohm" or simply "gyro-Bohm" scaling since the Bohm coefficient is reduced by $\rho_{*}$. The other extreme is the case where the magnetic configuration is completely stochastic and transport is along field lines. Then,

$$
\chi \propto a c_{\mathrm{s}} \propto \chi_{\mathrm{B}} \frac{a}{\rho_{\mathrm{s}}}
$$

indicating that $\chi$ is independent of $B$, which is the intuitive result. In this limit, $\alpha=-1$. Since it is not expected that transport can be any worse than this stochastic limit or any better than gyro-Bohm, these form the limits for reasonable values of $\alpha$. 
Empirical arguments lead to two intermediate values of $\alpha$. One is simply given by setting $\alpha=0$. This gives "Bohm" scaling because there is no longer any reduction of $\chi_{\mathrm{B}}$ by $\rho_{*}$. The original heuristic arguments about Bohm diffusion invoked an effective collision time due to turbulence which was proportional to the inverse of the cyclotron frequency [3]. Thus, the diffusive step of one gyroradius occurred in each gyroperiod. Modern heuristic arguments invoke turbulence which has a correlation length on the order of the machine size rather than the gyroradius; however, there has been little success theoretically in finding such modes. The other empirical argument arises from taking the confinement time to scale like $I / \sqrt{P}$, first introduced by Goldston [4]. Since this form is not dimensionally correct, it cannot be uniquely transformed to dimensionless variables; however, one reasonable transformation is to take the confinement time proportional to $1 / \beta q^{2}$. Since these two quantities are held fixed in this dimensionless scaling, the "Goldston" scaling along this path is $\chi$ independent of $B$ for fixed $a$. This implies that $\alpha=-1 / 2$ to cancel all the factors with $B$ in Eq. (1), when the scaling of $T$ with $B$ is taken into account.

In the DIII-D dimensionless scaling experiments, the size and shape of the plasma are held fixed and the magnetic field and temperature change to vary $\rho_{*}$ [5]. The dimensionless parameters used here are normalized gyroradius $\rho_{*} \equiv \rho / a, \beta \propto n T / B^{2}$, collisionality $\nu_{*} \propto n q / T^{2}$, and safety factor $q \propto B / I$, where $\rho_{*}, \beta$, and $\nu_{*}$ are defined separately for electrons and ions. Then for a change in $B$, the variables $n, T$, and $I$ must change like $n \propto B^{4 / 3}, T \propto B^{2 / 3}, I \propto B$ to keep $\beta, \nu_{*}$, and $q$ constant. The effective charge $Z_{\text {eff }}$, ion mass, the temperature ratio $T_{e} / T_{i}$, the heating profiles, and the density and temperature scale lengths should also be held constant. The variation in $\rho_{*}$ is proportional to $B^{-2 / 3}$. Because some of the discharges are heated with electron cyclotron heating (ECH), the toroidal field could only be varied by exactly a factor of two in order to preserve the heating profile. This leads to a variation in $\rho_{*}$ of a factor of 1.6. Table 1 gives the engineering parameters for the three cases which will be discussed in this paper - a low-density rf-heated pair of discharges, a high-density rf-heated pair, and a neutral beam injection (NBI) heated pair at a density close to the high-density rf case. Figure 1 shows the dimensionless quantities for the case $b$ versus the radial coordinate $r / a$, which is the square root of the enclosed toroidal flux normalized to the edge value. Over the region $0.4<r / a<0.8$, the dimensionless parameters are well matched and $\rho_{*}$ varies everywhere by the expected factor of 1.6 .

In order to determine the value of $\alpha$ for each of these cases, the ratio of the power balance diffusivities will be shown for both electrons and ions. To aid in understanding the relationship of $\alpha$ and the ratio of the diffusivities, Table 2 gives the expected ratio for the four cases discussed above: gyro-Bohm, Bohm, Goldston, and stochastic scaling. The scaling of the confinement time is just the inverse of that for $\chi_{\mathrm{DS}}$, as given by Eq. (3) for $a$ fixed. One major misconception surrounding this dimensionless scaling 
Table I

Engineering Parameters for Three Pair of Dimensionally Similar Discharges

\begin{tabular}{l|cc|cc|cc}
\hline & \multicolumn{2}{|c|}{$(\mathrm{a})$} & \multicolumn{2}{|c|}{ (b) } & \multicolumn{2}{c}{ (c) } \\
& ECH & ECH, FW & ECH & ECH, FW & NBI & NBI \\
\hline$B_{\mathrm{T}}(\mathrm{T})$ & 1.0 & 2.0 & 1.0 & 2.0 & 1.0 & 2.0 \\
$I_{\mathrm{p}}(\mathrm{MA})$ & 0.5 & 1.0 & 0.5 & 1.0 & 0.5 & 1.0 \\
$\bar{n}\left(10^{19} \mathrm{~m}^{-3}\right)$ & 1.0 & 2.6 & 1.4 & 3.8 & 1.2 & 3.1 \\
$W(\mathrm{~kJ})$ & 42 & 175 & 44 & 179 & 44 & 178 \\
$Z_{\text {eff }}$ & 3.2 & 2.3 & 2.5 & 2.1 & 3.0 & 1.6 \\
$P_{\text {tot }}(\mathrm{MW})$ & 1.1 & 2.2 & 0.6 & 2.0 & 0.6 & 1.9 \\
$\tau_{\mathrm{E}}(\mathrm{msec})$ & 39 & 81 & 74 & 91 & 74 & 93 \\
& & & & & & \\
\hline \hline
\end{tabular}

technique is that the scaling of $\tau_{E}$ with $B$ is the actual scaling which could be found by regression analysis of a database. This is clearly not true since we have enforced constraints on the relationship of $n, B, T$, and $I$ which are not preserved in a general database. The collisionality $\nu_{*}, \beta$, and $q$ all have $B$ dependences implicitly, and the $\rho_{*}$ scan has not determined the dependence of $\chi$ on these quantities. Therefore, the general $B$ dependence (and similarly, the general $T$ dependence or $n$ dependence) cannot be determined from a $\rho_{*}$ scan. In fact, this is exactly the point of the $\rho_{*}$ scan; the other dimensionless parameters need not change to extrapolate to a reactor, and the potentially complex dependencies on $n, T$, and $B$ need not be explicitly determined.

The ratio of the power balance diffusivities has been determined individually for electrons and ions for each of the three cases in Table 1. These ratios are plotted as a function of $r / a$ in Figs. 2(a), 3(a), and 4(a). In each case, the results are quite clear - the electrons scale like gyro-Bohm $(\alpha=1)$, while the ions scale as discussed above for Goldston scaling $(\alpha=-1 / 2)$. The error bars are determined by systematically varying the input data and combining the errors with the assumption that inputs are uncorrelated. The implication is that the difference in scaling between electrons and ions is not due to some onset in turbulence at high density, nor is it dependent on the heating source. Specifically, the ion $\rho_{*}$ scaling is the same in the case where the ions are only heated by collisional coupling (rf cases) and the case where power goes to the ions directly (NBI case). 

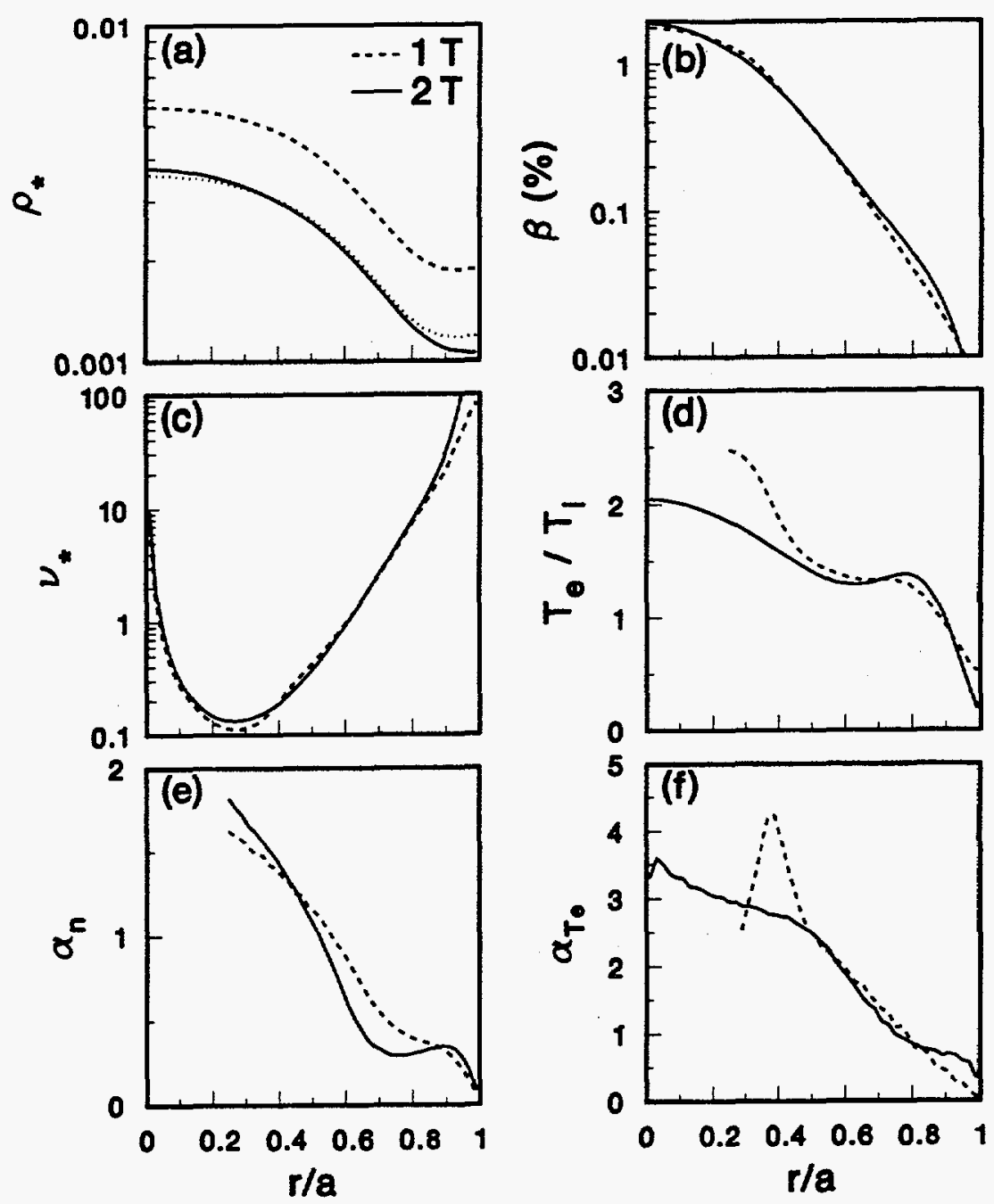

FIG. 1. Plots of dimensionless parameters versus the radial coordinate $r / a$ : (a) the ion normalized gyroradius (the dotted line is a factor of 1.6 change), (b) total beta, (c) electron collisionality, (d) temperature ratio, (e) density shape factor [defined as $\left(r^{2}-a^{2}\right) / 2 r L_{n}$ ], and (f) temperature shape factor (analogous to $\alpha_{n}$ ).

It is interesting to check what conclusions would have been drawn if an analysis based on effective diffusivity or global confinement time had been applied to the data, as was done in all previous dimensionless scaling experiments. Figures 2(a), 3(a), and 4 (a) show the ratio of the effective diffusivities defined by

$$
\chi_{\text {eff }} \equiv \frac{q_{e}+q_{\mathrm{i}}}{n_{\mathrm{e}} \nabla T_{\mathrm{e}}+n_{\mathrm{i}} \nabla T_{\mathrm{i}}}
$$


Table 2

Effective Scaling with $B$ and the Expected Ratio of the Power Balance Diffusivities for a Factor of Two Change in $B$

\begin{tabular}{llll}
\hline \hline & $\alpha$ & $\chi_{\mathrm{DS}}$ & $\chi_{2 T} / \chi_{1 T}$ \\
\hline gyro-Bohm & 1 & $B^{-1}$ & 0.5 \\
Bohm & 0 & $B^{-1 / 3}$ & 0.79 \\
Goldston & $-1 / 2$ & $B^{0}$ & 1.0 \\
stochastic & -1 & $B^{1 / 3}$ & 1.26 \\
\hline \hline
\end{tabular}

Also given in the figures is the ratio of the energy confinement times. By both measures, the low-density case has gyro-Bohm scaling, while the two high-density cases exhibit Bohm scaling. This is very reminiscent of the ohmic density scaling which has a transition from linear scaling to saturated at some density. The important point is that the analysis using the effective diffusivity can yield the spurious result that transport has Bohm scaling, when in fact neither species actually has Bohm scaling. To help understand why the scalings of the global confinement time and the effective diffusivity exhibit different scalings under different conditions while the individual species do not, the fractional power exhausted in the electron channel is plotted in Figs. 2(b), 3(b), and 4 (b) for the three cases, respectively. In the low-density of case (Fig. 2), all of the input power is to electrons, and the low density and high electron temperature reduce the collisional coupling to the ions. Therefore, most of the power is exhausted through electron conduction and the scalings of the global confinement and the effective diffusivity match the electron scaling. In the high-density rf case (Fig. 3), the input power is again exclusively in the electron channel. However, the collisional coupling is now much larger and roughly equal amounts of power are exhausted through electron and ion conduction. The scalings of the global confinement and the effective diffusivity are simply the weighted average of the individual scalings. The scaling appears as Bohm only by coincidence, not because of the intrinsic properties of the plasma. Likewise, the Bohm scaling of the confinement time and effective diffusivity in the NBI case (Fig. 4) are due to the weighted average of the electron and ion scaling. In this case the NBI puts power to both electrons and ions, and the collisional exchange term is a relatively minor part of the power balance. Clearly, global and one-fluid analysis can provide very misleading results. This may also explain why there has been no success in finding a theoretical model with Bohm scaling. 

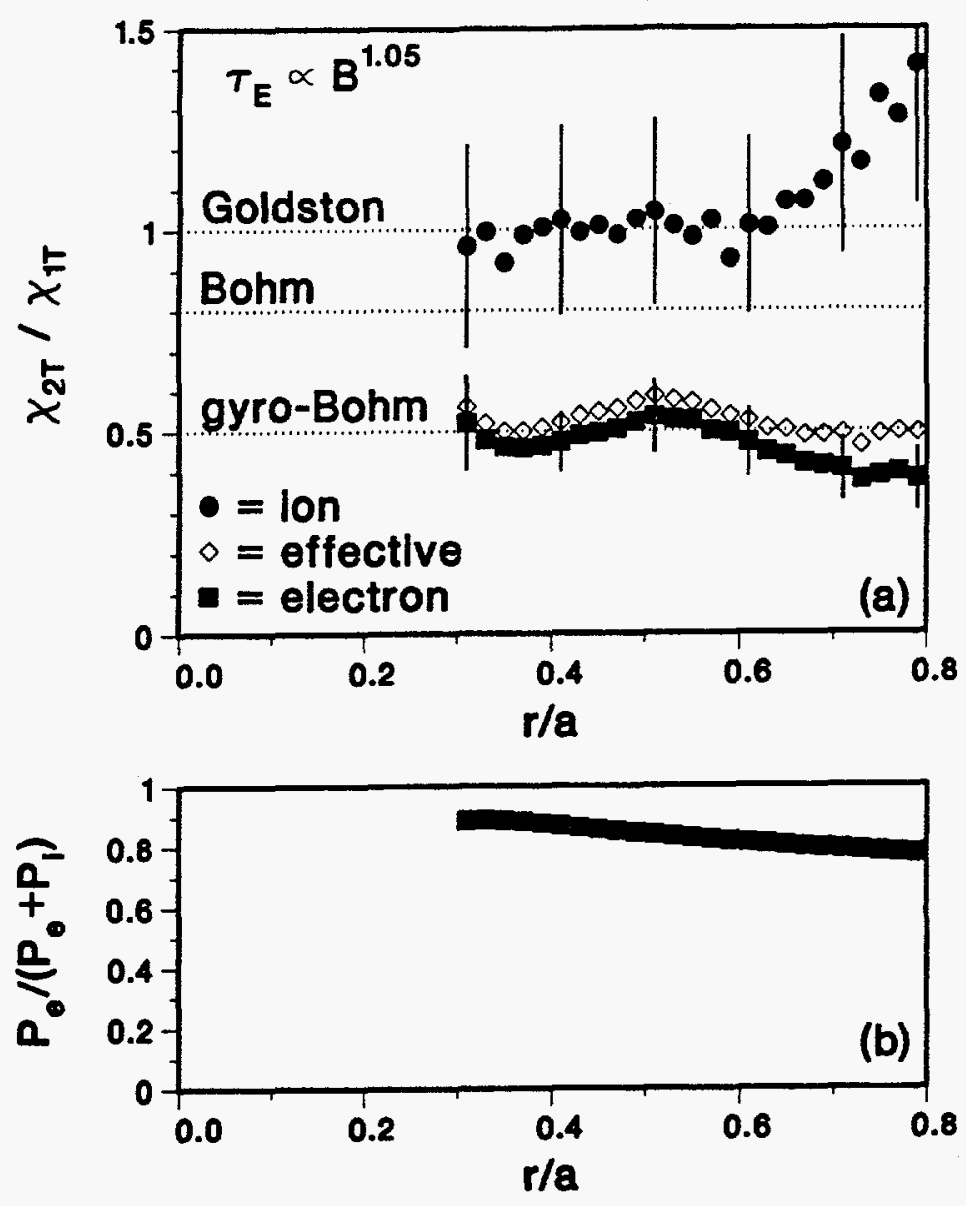

FIG. 2. (a) Ratio of the power balance diffusivities versus radius for the low-density rf-heated pair of discharges (case a in Table 1). Also shown is the effective diffusivity defined by Eq. (7). (b) The fractional power transported by electrons versus radius.

The observation of different $\rho_{*}$ scalings for electrons and ions can unify the various observations reported previously using analysis of the effective diffusivity. ECH experiments on DIII-D [6] and the Wendelstein VII-AS stellarator [7] both found gyro-Bohm scaling for the confinement time and the effective diffusivity. Since both experiments used electron heating at low density, the electron conduction was probably dominant as in Fig. 2. Ion cyclotron minority heating experiments on JET [8] and NBI experiments on TFTR [9] both found Bohm scaling for the total flux. The JET experiment was similar to the case shown in Fig. 3 with strong electron heating and strong electron-ion coupling. The TFTR experiment was more like the case shown in Fig. 4 with direct heating in both electrons and ions. Apparently, the uncertainty in the electron and ion diffusivities due to the strong exchange term was too large to make a two-fluid analysis 

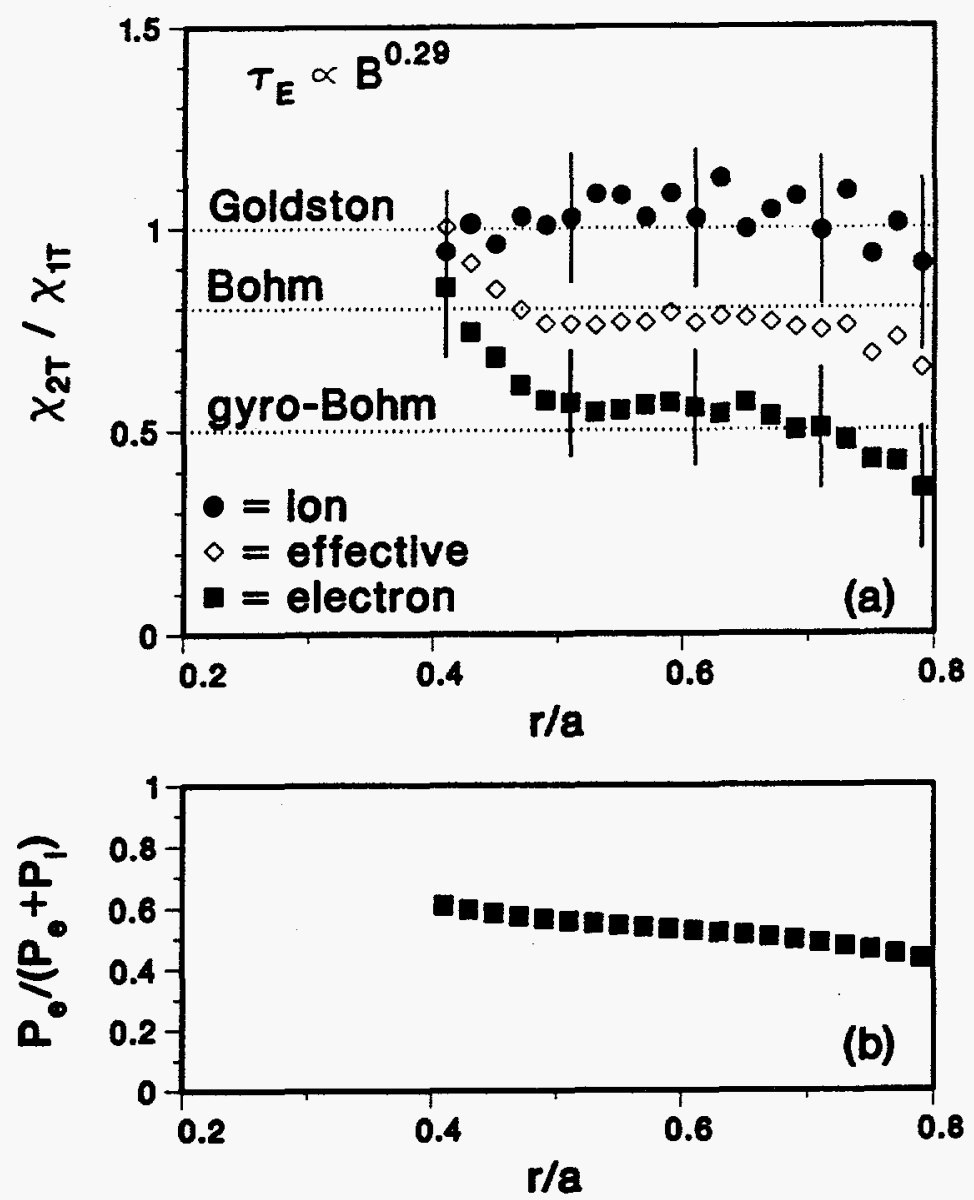

FIG. 3. (a) Ratio of the power balance diffusivities versus radius for the high-density rf-heated pair of discharges (case $b$ in Table 1). Also shown is the effective diffusivity defined in Eq. (7). (b) The fractional power transported by electrons versus radius.

in these cases. However, the observed $\rho_{*}$ scalings reported here for DIII-D can unify all of these previous experiments with reasonable assumptions.

The observation of separate ion and electron $\rho_{*}$ scalings may have a profound impact on the extrapolation to reactors such as ITER with smaller $\rho_{*}$ than present-day devices. First, it is important to acknowledge that the experiments reported here do not lie on a dimensionless scaling path to an attractive reactor. From Fig. 1, the $\beta$ is much too low and $\nu_{*}$ too high for a reasonable reactor. Also, $q$ is much higher than expected for a reactor, the ion mixture is not 50-50 D-T, the temperatures are not equilibrated, and the confinement is $\mathrm{L}$-mode rather than $\mathrm{H}$-mode. The effect of all these differences on the $\rho_{*}$ scaling is unknown. However, the $\rho_{*}$ scalings observed here make a startling prediction for devices at smaller $\rho_{*}$. If $Z_{\text {eff }}=1$ and $T_{\mathrm{e}} / T_{\mathrm{i}}=1$, the effective diffusivity can be written as 

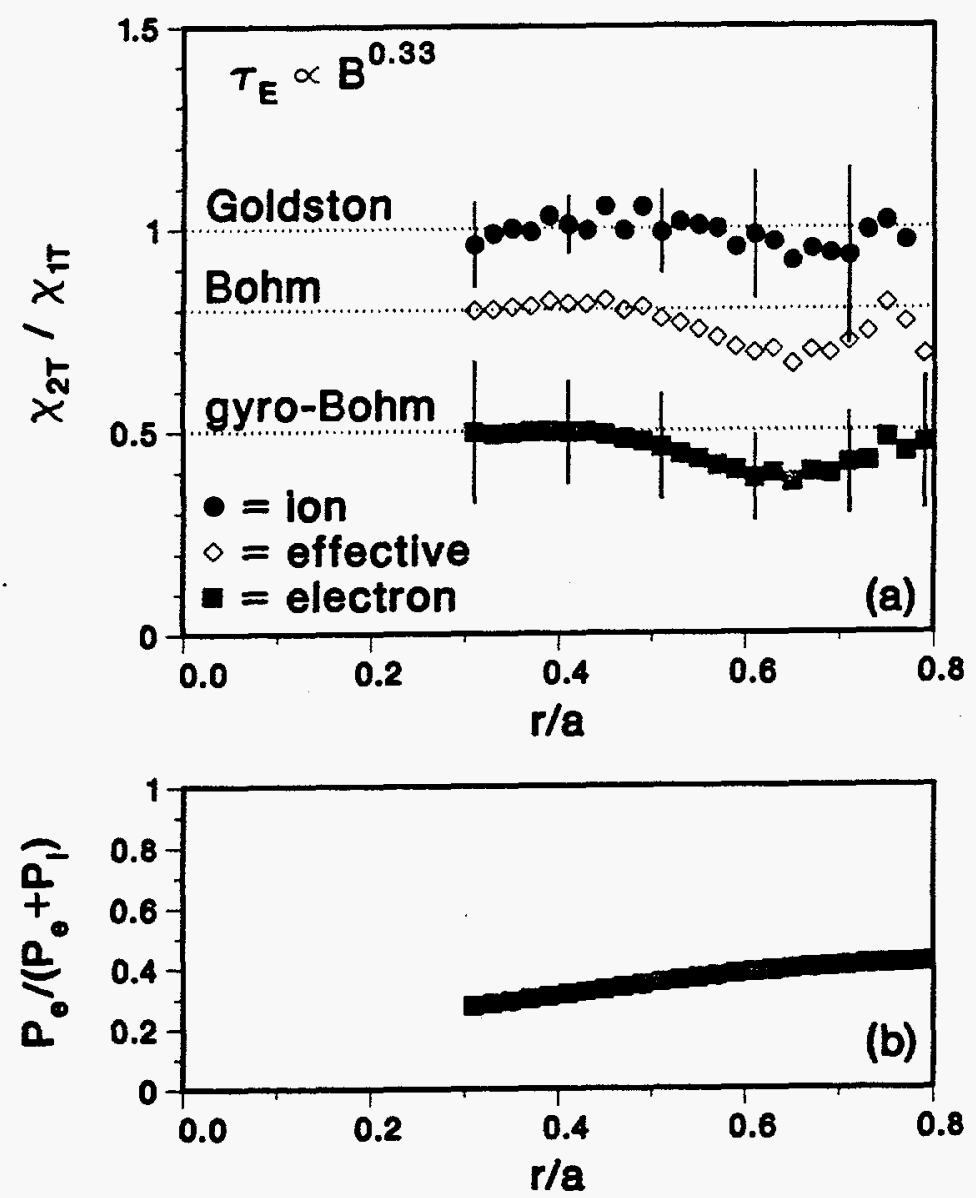

Fig. 4. (a) Ratio of the power balance diffusivities versus radius for the NBI-heated pair of discharges (case $c$ in Table 1). Also shown is the effective diffusivity defined in Eq. (7). (b) The fractional power transported by electrons versus radius.

$$
\chi_{\mathrm{eff}}=\frac{1}{2} \chi_{\mathrm{B}} \rho_{* e} F\left[1+\left(\frac{m_{\mathrm{i}}}{m_{\mathrm{e}}}\right)^{1 / 4} \frac{G}{F} \rho_{* e}^{-3 / 2}\right],
$$

where the $\rho_{*}$ scaling of the electrons is taken to be $\alpha=1$ and the ion scaling is taken to be $\alpha=-1 / 2$. The dependence of $\chi_{\text {eff }} / \chi_{\mathrm{B}}$ on $\rho_{*}$ is plotted in Fig. 5. At small $\rho_{*}$, the ion scaling dominates, while at large $\rho_{*}$, the scaling is gyro-Bohm like the electrons. This exercise clearly refutes the assertion that the scaling to ITER should be Bohm because that is what tokamaks with equilibrated temperatures observe. The fact that presentday large tokamaks with equilibrated temperatures observe Bohm scaling locates their position on the plot. Bohm scaling implies $\chi_{\text {eff }} / \chi_{B}$ is independent of $\rho_{*}$. The only place where this is true is at the minimum of the curve. The observed scaling implies that $\chi_{\text {eff }} / \chi_{B}$ will get rapidly larger when moving away from the present-day devices to 


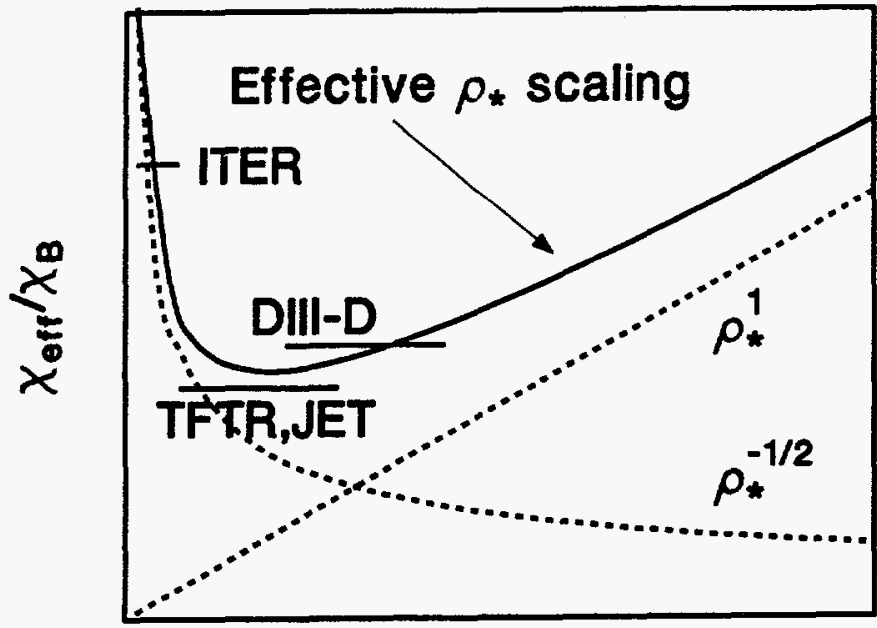

$$
\rho_{*, 0}
$$

FIG. 5. Plot of the $\rho_{*}$ dependence of the effective diffusivity using the observed values of $\alpha$ for the ions and electrons [Eq. (8)]. The dashed lines show the individual scalings of ions and electrons.

smaller $\rho_{*}$. Notice also that if the constraint of $T_{e}=T_{1}$ is released, this scaling permits the existence of hot-ion modes, if the ions are heated directly and decouple from the electrons. The $\chi_{\text {eff }} / \chi_{\mathrm{B}}$ will stay on the ion branch as it moves to larger $\rho_{*}$. Therefore, the observed $\rho_{*}$ scalings may also explain the asymmetry observed on JET in electron and ion heating [10].

In conclusion, the DIII-D data indicate distinct $\rho_{*}$ scalings for the two species - gyro-Bohm scaling for the electrons $\left(\chi \propto \chi_{\mathrm{B}} \rho_{*}\right)$ and Goldston scaling for the ions $\left(\chi \propto \chi_{\mathrm{B}} \rho_{*}^{-1 / 2}\right)$. This result is independent of the heating method. The global energy confinement time and the effective diffusivity scaling vary from gyro-Bohm to Bohm, depending on the fractional power exhausted in each channel. If this scaling persists at reactor-relevant values of $\beta, \nu_{*}$, and $q$ in a reactor-relevant geometry, the confinement is predicted to scale unfavorably with $\rho_{*}$ from present-day experiments.

The authors gratefully acknowledge the efforts of M.E. Austin, K.H. Burrell, S.C. Chiu, C.B. Forest, P. Gohil, J.S. deGrassie, R.A. James, R.J. La Haye, A.W. Leonard, R.I. Pinsker, and D. Wròblewski in obtaining and analyzing the data, and useful discussions with R.E. Waltz on the conceptual groundwork of dimensionless scaling.

This is a report of work sponsored by the U.S. Department of Energy under Contract No. DE-AC03-89ER51114. 


\section{References}

[1] B.B. Kadomtsev, Sov. J. Plasma Phys. 1, 295 (1975).

[2] J.W. Connor and J.B. Taylor, Nucl. Fusion 17, 1047 (1977).

[3] N.A. Krall and A.W. Trivelpiece, Principles of Plasma Physics (McGraw-Hill, 1973).

[4] R.J. Goldston, Plasma Phys. and Contr. Fusion 26, 87 (1984).

[5] C.C. Petty, T.C. Luce, R.I. Pinsker, et al., Gyroradius Scaling of Electron and Ion Transport, General Atomics Rep. GA-A21632 (1994), submitted to Phys. Rev. Lett.

[6] C.C. Petty, T.C. Luce, and R.I. Pinsker, in Radio-Frequency Power in Plasmas: 10th Top. Conf. 1993, Boston (American Institute of Physics, New York, 1994), p. 165.

[7] U. Stroth et al., Phys. Rev. Lett. 70, 936 (1993).

[8] J.P. Christiansen et al., Nucl. Fusion 33, 863 (1993).

[9] F. W. Perkins, Jr. et al. Phys. Fluids B 5, 477 (1993).

[10] JET Team, in Plasma Physics and Controlled Nuclear Fusion Research 1990 (Proc. 13th Int. Conf. Washington, DC, 1990) (IAEA, Vienna, 1991), Vol. 1, p. 27. 